\title{
BMJ Open Unusually low prevalence of Mycoplasma genitalium in urine samples from infertile men and healthy controls: a prevalence study
}

\author{
Vanda Plecko, ${ }^{1}$ Lidija Zele-Starcevic, ${ }^{1}$ Vesna Tripkovic, ${ }^{1}$ Mihael Skerlev, ${ }^{2}$ \\ Suzana Ljubojevic, ${ }^{2}$ Sanja Plesko, ${ }^{1}$ Ivana Marekovic, ${ }^{1}$ Jorgen Skov Jensen ${ }^{3}$
}

To cite: Plecko V, ZeleStarcevic L, Tripkovic V, et al. Unusually low prevalence of Mycoplasma genitalium in urine samples from infertile men and healthy controls: a prevalence study. BMJ Open 2014;4:e005372.

doi:10.1136/bmjopen-2014005372

- Prepublication history for this paper is available online. To view these files please visit the journal online (http://dx.doi.org/10.1136/ bmjopen-2014-005372).

Received 31 March 2014 Revised 14 July 2014 Accepted 1 August 2014

\section{(1) CrossMark}

${ }^{1}$ Department of Clinical and Molecular Microbiology, Clinical Hospital Centre Zagreb and School of Medicine University of Zagreb, Zagreb, Croatia 2Department of Dermatology and Venerology, Clinical Hospital Centre Zagreb and School of Medicine University of Zagreb, Zagreb, Croatia ${ }^{3}$ Mycoplasma Laboratory, Staten's Serum Institute, Copenhagen, Denmark

Correspondence to Dr Vesna Tripkovic; vbtripkovic@gmail.com

\section{ABSTRACT}

Objective: To detect Mycoplasma genitalium in urine samples of infertile men and men without any signs of infection in order to investigate whether $M$. genitalium and other genital mycoplasmas (Mycoplasma hominis and Ureaplasma spp) are found more often in urine samples of infertile men than in asymptomatic controls and to determine resistance to macrolides.

Methods: The study included first void urine samples taken from 145 infertile men and 49 men with no symptoms of urethritis. M. genitalium, Chlamydia trachomatis and Neisseria gonorrhoeae were detected by commercial PCR. Trichomonas vaginalis was detected by microscopy and culture. M. hominis and Ureaplasma spp were detected by culture. $M$. genitalium was detected by in-house conventional and real-time PCR.

Results: Two M. genitalium positive samples were found among samples obtained from infertile men. All asymptomatic men were $M$. genitalium negative.

Macrolide resistance was not found in either of the two positive samples.

Conclusions: In comparison with reported data, an unusually low prevalence of $M$. genitalium was found in infertile men. The reasons for this unexpected result are not known; possibly, local demographic and social characteristics of the population influenced the result. Further studies to investigate $M$. genitalium in infertile and other groups of patients are needed.

\section{INTRODUCTION}

Reliable detection of Mycoplasma genitalium became possible after the development of PCR assays. ${ }^{2}$ The prevalence of M. genitalium in patients with non-gonococcal urethritis (NGU) ranges from $13 \%$ to $42 \%$; and in asymptomatic men from $0 \%$ to $15 \% .{ }^{2}-{ }^{4}$ The impact of $M$. genitalium on male fertility remains unclear. ${ }^{25}$

Our aim was to detect $M$. genitalium, $M$. hominis and Ureaplasma spp in first void urine (FVU) samples of infertile men and men

\section{Strengths and limitations of this study}

We are aware of low number of urine samples in both groups of participants.

- These are only preliminary results, and were unexpected in comparison with reported data. However, the results were confirmed in two independent laboratories to exclude the possibility of laboratory error.

- We plan to continue this study, collecting urine samples from a larger number of infertile men and comparing them both with men with urethritis and asymptomatic men.

- If the results are similar (ie, an unexpectedly small number of men with Mycoplasma genitalium), we will try to determine the reason for this.

without any symptoms and/or signs of infection and, additionally, to determine the prevalence of macrolide resistance in $M$. genitalium. We restricted our study to infertile men without the following common sexually transmitted infections (STIs): Chlamydia trachomatis, Trichomonas vaginalis and Neisseria gonorrhoeae.

To our knowledge, this is the first study in Croatia which has been undertaken to detect M. genitalium.

\section{METHODOLOGY}

The study was approved by the ethics committee of the School of Medicine, University of Zagreb. It is part of the Croatian Ministry of Science grant (108-1080114-0014): "Molecular detection of microorganisms: their influence on antimicrobial consumption".

Each participant provided written informed consent, and completed a questionnaire stating reasons for attendance, age, symptoms of urethritis, number of lifetime 
sexual partners, history of STIs and recent/current antibiotic treatment.

FVU (about $20 \mathrm{~mL}$ ) was taken from the patients; 4-5 mL of each sample was used for culture of $M$. hominis, ureaplasmas and T. vaginalis. For PCR detection of M. genitalium, C. trachomatis and N. gonorrhoeae, 4-5 mL of each sample were used. Five millilitres of the original FVU were frozen and shipped to Staten Serum Institut in Copenhagen for confirmation by real-time $M$. genitalium PCR. Samples were immediately processed for M. hominis, T. vaginalis and ureaplasmas. For PCR detection samples were stored at $-20^{\circ} \mathrm{C}$.

C. trachomatis and N. gonorrhoeae were detected by PCR (Cobas TaqMan CT/NG Test, V.2.0 Roche Diagnostic, Basel, Switzerland), as described by the manufacturer, and urethral swabs were obtained for detection of $N$. gonorrhoeae by culture (BBL MTM, New Jersey, USA). T. vaginalis was detected by microscopy and culture in modified Diamonds medium (Remel, Inc, Santa Fe, USA). Thirteen of the infertile men and three controls were excluded owing to infection with a recognised STI (Chlamydia trachomatis, Trichomonas vaginalis and Neisseria gonorrhoeae), leaving a total of 194 FVU samples. These were collected in polypropylene containers (Sarstedt, Nümbrecht, Germany) from men who were referred to the Department of Clinical Microbiology and Department of Dermatology, Clinical Hospital Centre Zagreb. One hundred and forty-five samples were obtained from men as a part of an infertility investigation. None of them had any symptoms of genitourinary infections, but all had an abnormal semen quality analysis (oligozoospermia, asthenozoospermia, oligoasthenozoospermia). Infertility of their female partners was excluded by hysterosonosalpingography.

Forty-nine samples were from asymptomatic men attending the clinic as a part of an annual physical examination.

Aliquots of the urine samples $(4-5 \mathrm{~mL})$ were prepared for culture of genital mycoplasmas and for molecular testing, respectively. Urine samples were centrifuged at $3000 \times g$ for $5 \mathrm{~min}$ and sediments were inoculated in urea-arginine broth (bioMerieux, Lyon, France) and onto A7 agar (Becton Dickinson, Cockeysville, Maryland, USA). The vials were incubated for $48 \mathrm{~h}$ at $37^{\circ} \mathrm{C}$. The agar plates were incubated at $37^{\circ} \mathrm{C}$ in $5 \% \mathrm{CO}_{2}$ for 5 days and examined microscopically for the appearance of typical mycoplasma colonies. ${ }^{6}$

The rest of the urine $(4-5 \mathrm{~mL})$ was concentrated at $20000 \times \mathrm{g}$ for $15 \mathrm{~min}$ at $4^{\circ} \mathrm{C}$. The pellet was resuspended in $200 \mu \mathrm{L}$ of $20 \% \mathrm{w} / \mathrm{v}$ Chelex 100 slurry (Sigma, USA) in TE buffer (10 mM Tris-HCl, pH 8.0, 1 mM EDTA). The mixture was vortexed for $1 \mathrm{~min}$; then placed in a thermoblock for $10 \mathrm{~min}$ at $95^{\circ} \mathrm{C}$. The mixture was centrifuged briefly and the supernatant aspirated into new tubes and stored at $-20^{\circ} \mathrm{C}$ until required for PCR. ${ }^{7}$

PCR for detection of $M$. genitalium was performed with two pairs of primers: the first targeted the $16 \mathrm{~S}$ rRNA gene: 16SFG2 (5'-CCT TAT CGT TAG TTA CAT
TGT TTA A), 16SRG (5'-TGA CAT GCG CTT CCA ATA AA), ${ }^{7}$ and the second targeted the MgPa major adhesin gene: MgPal (5'-AGT TGA TGA AAC CTT AAC CCC TTG G), MgPa3 (5'-CCG TTG AGG GGT TTT CCA TTT TTG C). ${ }^{1}$

All samples were examined by both assays with internal controls for PCR inhibition, and both PCRs were performed as previously described. ${ }^{17-9}$

The PCR was performed in an automated DNA thermal cycler (PCR System 9700, Applied Biosystems).

To confirm the results, an aliquot of the original FVU samples (5 mL) was shipped to Statens Serum Institut, Copenhagen, Denmark, where it was tested by an inhibitor-controlled real-time PCR using primers detecting the MgPa gene, as previously described. ${ }^{79}$

Macrolide resistance mediating mutations in region $\mathrm{V}$ of the 23S rRNA gene was detected by DNA sequencing of amplicons obtained directly from the clinical specimens, and performed at Statens Serum Institute Copenhagen, Denmark. ${ }^{10}$

STATISTICA (data analysis software system), V.10 (StatSoft, Inc (2011), USA) was used for data analysis. The median was used to describe the age of groups and the number of observations and percentage to describe categorical variables. To compare age between the groups a Mann-Whitney $\mathrm{U}$ test was used, and to compare categorical variables a $\chi^{2}$ test was used. A $\mathrm{p}$ value of $<0.05$ was considered to be statistically significant for all tests performed.

\section{RESULTS}

The infertile men were comparable to the controls for age $(\mathrm{z}=-0.805, \mathrm{p}=0.421$, Mann-Whitney $\mathrm{U}$ test). They significantly more often reported a history of STIs $\left(\chi^{2}=14.443, \mathrm{df}=1, \mathrm{p}=0.0001\right)$ and a higher number of lifetime sexual partners $\left(\chi^{2}=35.734, \mathrm{df}=2, \mathrm{p}<0.0001\right.$; table 1$)$.

Thirteen of the infertile men and three controls were excluded owing to infection with a recognised STI (Chlamydia trachomatis, Trichomonas vaginalis and Neisseria gonorrhoeae).

\begin{tabular}{|c|c|c|}
\hline Data & $\begin{array}{l}\text { Infertile men } \\
(\mathrm{N}=145)\end{array}$ & $\begin{array}{l}\text { Asymptomatic men } \\
(\mathrm{N}=49)\end{array}$ \\
\hline $\begin{array}{l}\text { Age, median } \\
\text { (years) }\end{array}$ & 38 & 41 \\
\hline History of STIs* & $81(55.8)$ & $12(24.4)$ \\
\hline \multicolumn{3}{|c|}{ No of lifetime partners $†$} \\
\hline$<5$ & 15 (10.3) & $13(26.5)$ \\
\hline $5-10$ & $123(84.8)$ & $25(51.0)$ \\
\hline$>10$ & $7(4.8)$ & $11(22.4)$ \\
\hline \multicolumn{3}{|c|}{$\begin{array}{l}\text { Results are shown as number }(\%) \text { unless stated otherwise. } \\
{ }^{*} p=0.0001, \chi^{2} \text { test; } \\
t p<0.0001, \chi^{2} \text { test. } \\
\text { STIs, sexually transmitted infections. }\end{array}$} \\
\hline
\end{tabular}


Among the infertile men one patient had N. gonorrhoeae infection, one patient was diagnosed with T. vaginalis and 11 patients were C. trachomatis positive. These samples were also tested for $M$. genitalium, but all were negative.

Ureaplasma spp and M. hominis were isolated from the same proportion of infertile men and asymptomatic controls $\left(\chi^{2}=0.435, \mathrm{p}=0.509 ; \chi^{2}=0.021, \mathrm{p}=0.886\right)$, respectively. Only two samples were positive for M. genitalium; both in the group of infertile men $(2 / 145 ; 1.4 \% ; 95 \%$ CI $0.2 \%$ to $4.9 \%$ ). These men were aged 29 and 37 years, respectively, and reported three and seven lifetime sexual partners compared with the majority of the group who had had 5-10 partners.

In our laboratory we used conventional in-house PCR (qualitative) and results were confirmed at the Staten Serum Institut in Copenhagen, Denmark by real-time PCR. All M. genitalium results were concordant when the samples were examined by real-time PCR in Copenhagen. M. genitalium load for two positive samples was 778 copies $/ \mathrm{mL}(\mathrm{c} / \mathrm{mL})$ and $6765 \mathrm{c} / \mathrm{mL}$, respectively. The man with a $M$. genitalium load of $778 \mathrm{c} / \mathrm{mL}$ was diagnosed with oligozoospermia, and the other (M. genitalium load of $6765 \mathrm{c} / \mathrm{mL}$ ) with asthenozoospermia.

Ureaplasma spp were found in $30 \%(43 / 145)$ of the infertile men compared with $35 \%(17 / 49)$ of the asymptomatic men. M. hominis was positive in $21 \%(31 / 145)$ and $20 \%(10 / 49)$ of infertile men and asymptomatic men, respectively.

In 12 samples from the infertile men co-infection with Ureaplasma spp. and M. hominis was found. In the group of asymptomatic men co-infection in three samples was found. In the two samples with positive M. genitalium, taken from infertile men, no other pathogens were present.

Macrolide resistance mediating mutations in the 23S rRNA gene of $M$. genitalium were not found in either of the two positive samples.

\section{DISCUSSION}

This study demonstrates a low prevalence of M. genitalium in infertile men in the Zagreb region, Croatia. Ureaplasmas and M. hominis were often detected in both infertile men and healthy controls, suggesting that they should be considered commensals.

FVU samples were used because several studies have reported that molecular methods performed on urine samples can detect as many, or even more, infected patients than traditional urethral swabs, or cervical swabs or semen. ${ }^{57}$ No data for the prevalence of genital mycoplasmas in Croatia exist.

In this study the prevalence of Ureaplasma spp did not differ significantly among infertile men and asymptomatic controls, and was present in about one-third of both groups. This strongly suggests that ureaplasmas do not have a significant role of in male infertility.

We did not perform a specific test for the Ureaplasma spp. Most of the published studies have reported the prevalence of ureaplasmas in infertile men without discriminating between $U$. urealyticum and $U$. paroum. The data are not conclusive about the prevalence of $U$. urealyticum and $U$. parvum. Abusaraha et al, ${ }^{11}$ found that $U$. parvum was the most prevalent isolate detected among infertile men $(90 \%)$.

M. hominis was detected in $20 \%$ of asymptomatic men and $21 \%$ of infertile men, respectively, a higher prevalence than in some other studies. ${ }^{12} M$. hominis is considered normal flora of the urethra and the prevalence of $M$. hominis may reflect a high prevalence of bacterial vaginosis in the men's sexual partners, as M. hominis is known to be strongly associated with this condition in women. $^{13}$

The prevalence of $M$. genitalium varies significantly in different populations ${ }^{2}$ and was low in our study. Other studies have also found that M. genitalium is uncommon in the FVU of infertile men. ${ }^{5}$ The two positive samples in our study were from the group of 145 infertile men and M. genitalium was not detected in any of the controls. We were concerned that technical problems might have caused the low prevalence, and therefore, frozen FVU samples were shipped to Copenhagen for evaluation. However, a $100 \%$ concordance between the results was found, suggesting that the prevalence of M. genitalium in this Croatian population is, indeed, very low.

A possible relationship between infection and infertility has been the subject of controversy for years. It is estimated that only $15 \%$ of male infertility is related to genital tract infection. ${ }^{5}{ }^{14}$ Detection of bacteria in urogenital samples does not necessarily suggest infection but may signify colonisation, contamination or infection.

Only a few studies have examined the association between $M$. genitalium and male infertility ${ }^{5} 15$ and these studies did not have control populations of fertile men. We tried to design a study in which all other potential infective causes of infertility were excluded. In recent studies the prevalence of $M$. genitalium in infertile men was similar to the prevalence found by us (1.4\%): $4.8 \%$ in the study of Gdoura $e t a \tilde{l}$ and $3.2 \%$ was in the study of Abusaraha $e t a l^{11}$

Findings for C. trachomatis, which is the most common bacterial cause of NGU, were similar. C. trachomatis in women is a well-established cause of tubal factor infertility; in men it causes NGU. Also, it has been shown that C. trachomatis attaches to spermatozoa (on the surface and in the nucleolus). However, its role in male infertility, like the role of $M$. genitalium, is not yet clear. There are significant variations in the prevalence of C. trachomatis infections in men with infertility ranging from $0 \%$ to $42.3 \%$, depending on the methodology, type of sample and differences of infection rates in different populations. In a recently published Canadian study ${ }^{16}$ the prevalence of C. trachomatis infection in 5588 infertile men, was $0.3 \%$. The author concluded that this low prevalence clearly demonstrates that a small proportion of male infertility is caused by C. trachomatis. ${ }^{17}$

We attempted to study an asymptomatic group of men without urethritis as controls, and found that all were 
negative for $M$. genitalium. Unfortunately, the infertile men had had significantly more partners and also reported previous STIs more commonly than did the controls, suggesting that the control group had less risky behaviour.

Both M. genitalium positive samples were tested for macrolide resistance and were susceptible. This is encouraging considering the widespread use of azithromycin in the treatment of chlamydia and unspecific urethritis in Croatia. It is not possible to provide estimates of the prevalence of resistance to macrolides in this bacterium. Obviously, more $M$. genitalium positive samples should be tested in order to guide future treatment guidelines.

An unusually low percentage of $M$. genitalium was found in this study. The reasons for this unexpected result cannot be explained. Further studies to investigate M. genitalium in infertile and other group of patients from Croatia are needed.

Acknowledgements We thank Birthe Dohn for excellent technical assistance with M. genitalium testing at Statens Serum Institut, Copenhagen, Denmark.

Contributors VP designed the study, was responsible for questionnaire data and data analysis, and wrote part of the manuscript; LZ-S tested samples for Mycoplasma genitalium, and drafted the article; VT planned the study and wrote part of the text; MS collected samples and edited the manuscript; SL collected samples and questionnaire data; JSJ tested samples for $M$. genitalium, edited the manuscript and approved the final version; SP tested samples and corrected the draft version; IM revised the manuscript.

Funding The study is part of the Croatian Ministry of Science grant (108-1080114-0014): "Molecular detection of microorganisms: their influence on antimicrobial consumption".

Competing interests None.

Patient consent Obtained.

Ethics approval Ethics committee, School of Medicine, University of Zagreb.

Provenance and peer review Not commissioned; externally peer reviewed.

Data sharing statement No additional data are available.

Open Access This is an Open Access article distributed in accordance with the Creative Commons Attribution Non Commercial (CC BY-NC 4.0) license, which permits others to distribute, remix, adapt, build upon this work noncommercially, and license their derivative works on different terms, provided the original work is properly cited and the use is non-commercial. See: http:// creativecommons.org/licenses/by-nc/4.0/

\section{REFERENCES}

1. Jensen JS, Uldum SA, Sondergard-Andersen J, et al. Polymerase chain reaction for detection of Mycoplasma genitalium in clinical samples. J Clin Microbiol 1991;29:46-50.

2. Taylor-Robinson D, Jensen JS. Mycoplasma genitalium: from chrysalis to multicolored butterfly. Clin Microbiol Rev 2011;24:498-514

3. Ross JDC, Jensen JS. Mycoplasma genitalium as a sexually transmitted infection: implications for screening, testing, and treatment. Sex Transm Infect 2006;82;269-71.

4. Ishihara S, Yasuda M, Ito S, et al. Mycoplasma genitalium urethritis in men. Int J Antimicrobial Agents 2004;24S:S23-7.

5. Gdoura R, Kchaou W, Ammar-Keskes L, et al. Assessment of Chlamydia trachomatis, Ureaplasma urealyticum, Ureaplasma parvum, Mycoplasma hominis, and Mycoplasma genitalium in semen and first void urine specimens of asymptomatic male partners of infertile couples. J Androl 2008;29:198-206.

6. Shepard MC, Lunceford CD. Differential agar medium (A7) for identification of Ureaplasma urealyticum (human T mycoplasmas) in primary cultures of clinical material. J Clin Microbiol 1976;3:613-25.

7. Jensen JS, Björnelius E, Dohn B, et al. Comparison of first void urogenital swab specimens for detection of Mycoplasma genitalium and Chlamydia trachomatis by polymerase chain reaction in patients attending a sexually transmitted disease clinic. Sex Transm Dis 2004;31:499-507.

8. Eastick K, Leeming JP, Caul EO, et al. A novel polymerase chain reaction assay to detect Mycoplasma genitalium. Mol Pathol 2003;56:25-8.

9. Jensen JS, Björnelius E, Dohn B, et al. Use of TaqMan $5^{\prime}$ nuclease real-time PCR for quantitative detection of Mycoplasma genitalium DNA in males with and without urethritis who were attendees at a sexually transmitted disease clinic. J Clin Microbiol 2004;42:683-92.

10. Jensen JS. Protocol for the detection of Mycoplasma genitalium by PCR from clinical specimens and subsequent detection of macrolide resistance-mediating mutations in region $\mathrm{V}$ of the 23S rRNA gene. Methods Mol Biol 2012;903:129-39.

11. Abusaraha EA, Awwadb ZM, Charvalosc E, et al. Molecular detection of potential sexually transmitted pathogens in semen and urine specimens of infertile and fertile males. Diagn Microbiol Infect Dis 2013;77:283-6.

12. Salmeri M, Valenti D, La Vignera S, et al. Prevalence of Ureaplasma urealyticum and Mycoplasma hominis infection in unselected infertile men. J Chemother 2012;24:81-6.

13. Totten PA, Taylor-Robinson D, Jensen JS. Genital mycoplasmas. In: Holmes KK, Sparling PF, Stamm WE, et al., eds. Sexually transmitted diseases. 4 edn. New York: McGraw Hill, 2008:709-36.

14. Svenstrup HF, Fedder J, Abraham-Peskir J, et al. Mycoplasma genitalium attaches to human spermatozoa. Hum Reprod 2003:18:2103-9.

15. Gdoura R, Kchaou W, Chaari C, et al. Ureaplasma urealyticum Ureaplasma parvum, Mycoplasma hominis and Mycoplasma genitalium infections and semen quality of infertile men. BMC Infect Dis 2007;7:129.

16. Samplaski MK, Domes T, Jarvi KA. Chlamydial infection and its role in male infertility. Adv Androl 2014:2014,307950, 11 pages http://dx. doi.org/10.1155/2014/307950

17. Domes T, Lo KC, Grober ED, et al. The utility and cost of Chlamydia trachomatis and Neisseria gonorrhoeae screening of a male infertility population. Fertil Steril 2012;97:299-305. 